\title{
Caracterización de las estrategias de la enseñanza universitaria y la actitud del profesorado hacia la innovación en tiempos de pandemia
}

\author{
Lida Valenzuela \\ lidavalenzuea@gmail.com \\ Facultad de Humanidades y Ciencias de la Educación \\ Universidad Nacional de Pilar \\ Cresencia Barrios \\ cprantte2012@gmail.com \\ Facultad de Humanidades y Ciencias de la Educación \\ Universidad Nacional de Pilar
}

\section{RESUMEN}

El trabajo analiza las estrategias aplicadas en la enseñanza universitaria y la actitud de los docentes hacia la innovación en tiempos de pandemia, pretende describir las acciones de enseñanza en el marco de una educación de calidad de la Facultad de Humanidades y Ciencias de la Educación de la Universidad Nacional de Pilar, Paraguay. El abordaje es necesario considerando el tiempo de crisis instalado a raíz de la pandemia mundial que obliga a las instituciones de educación superior realizar ajustes a las estrategias de enseñanza y aprendizaje. La metodología adoptó el enfoque cuantitativo, con diseño observacional; prospectivo; transversal; descriptivo. La población de estudio lo conformaron el $100 \%$ de los docentes de cuatro carreras universitarias y se recurrió como técnica de recolección de los datos a la encuesta, a través de un instrumento, y la observación documental: El análisis de los resultados permitió la caracterización de la enseñanza universitaria y la actitud del docente hacia la innovación.

Palabras-clave: transformación educativa, enseñanza universitaria, actitud, innovación. 


\title{
Characterization of university teaching strategies and the attitude of teachers towards innovation in times of pandemic
}

\begin{abstract}
ABSTRAC
The work analyzes the strategies applied in university and the attitude of teachers towards innovation in times of pandemic, it aims to describe the teaching actions within the framework of a quality education of the Faculty of Humanities and Education Sciences of the National University of Pilar, Paraguay. The approach is necessary considering the time of crisis as a result of the global pandemic that forces higher education institutions to make adjustments to teaching and learning strategies. The methodology adopted the quantitative approach, with an observational design; prospective; cross; descriptive. The study population was made up of $100 \%$ of the teachers of four university careers and the survey was used as a data collection technique, through an instrument, and documentary observation: The analysis of the results allowed the characterization of university teaching and the teacher's attitude towards innovation
\end{abstract}

Keywords: educational transformation, university teaching, attitude, innovation

Artículo recibido: 10 oct. 2020

Aceptado para publicación: 14 nov. 2020

Correspondencia:lidavalenzuea@gmail.com.

Conflictos de Interés: Ninguna que declarar 


\section{INTRODUCCIÓN}

La pandemia del coronavirus está cambiando instantáneamente la forma en que se imparte la educación, la Facultad, el colegio o la escuela y el hogar ahora se convierten en el mismo lugar tras las necesarias regulaciones efectuadas. Según la UNESCO, más de 861.7 millones de niños y jóvenes en 119 países se han visto afectados al tener que hacer frente a la pandemia global que nos ha sacudido este año. Millones de familias en EE.UU. se han tenido que unir al 1.7 millón de niños que se encuentran enrolados en la educación en el hogar (homeschooling) (Villafuerte, 2020).

En este contexto, la Educación Superior es objeto de amplios debates desde múltiples aristas, en los cuales se busca predecir sus tendencias futuras para nuestra región, debido que particularmente en los países de América Latina, se ha transitado por un amplio y dinámico conjunto de cambios en los sistemas educativos, especialmente en el nivel superior. (Villalba Benítez, 2017).

Ya desde la declaración final de la CRES 2018, (Conferencia Regional de la Educación Superior), destaca que las universidades deben comprometerse activamente con la transformación social, económica y tecnológica de los países de la región, estableciéndose un Plan de Acción que debe ser articulada y concretada. Como una de las acciones del plan se propone, "reivindicar la necesaria transformación de las funciones sustantivas de las instituciones de educación superior desde la responsabilidad social con la más plena vigencia de la formación humanista e integral de las personas" (Henríquez Guajardo, 2018, p.118).

Es así que en cuestión de semanas, se ha cambiado la manera en la que los estudiantes aprenden, y justo estas transformaciones permiten observar las fallas en materia de equidad que sigue presentando el sistema educativo, incluso en los círculos más privilegiados. El World Economic Forum, plantea que esta pandemia se convierte en una oportunidad para recordarnos las habilidades que nuestros estudiantes necesitan justo en crisis como estas, siendo así, la toma de decisiones informada, resolución creativa de problemas y, sobre todo, adaptabilidad. Para garantizar que esas habilidades sigan siendo una prioridad para todos los alumnos, la resiliencia también debe integrarse en nuestros sistemas educativos. (Villafuerte, 2020)

En este sentido, aspectos muy importantes fueron planteados ya en el Foro Internacional Vanguardia en la Educación 2019, (Wolpert, 2019), donde se remarcó que para lograr 
la Agenda 2030 para el Desarrollo Sostenible,( sin saber aún la crisis de la pandemia) es necesario propiciar pensamientos y habilidades transformadoras, innovadoras y creativas que permitan avanzar hacia un desarrollo sostenible, además de forjar las habilidades para convertir a los estudiantes en ciudadanos empoderados que participen en la atención de los problemas que hoy en día afectan a los países y las regiones de todo el planeta.

En esta investigación se aborda el rol primordial del docente en la Educación Superior, específicamente las estrategias de enseñanza y la actitud necesaria para la innovación en tiempos de pandemia, con miras a la tan anhelada calidad educativa, asumiendo que la educación es uno de los motores más poderosos y probados para garantizar el desarrollo sostenible de los países en desarrollo como Paraguay, contemplados en los diversos documentos regionales y nacionales mencionados.

Cabe resaltar que el Estado paraguayo cuenta con una norma vigente, que es la Ley 4995 de Educación Superior, además asume las declaraciones de organismos internacionales suscriptos, como la CRES y UNESCO (2009) que reconocen la Educación Superior como un bien público y un derecho humano fundamental. El Estado garantizará el derecho a aprender, la igualdad de oportunidades $\sin$ discriminación alguna, establece la Constitución Nacional de 1992 (Villalba, 2017).

En este marco de referencia, se especifica que en Paraguay, el nivel de educación superior está compuesto por 9 Universidades Públicas, 54 Universidades Privadas, 34 Institutos Superiores, 127 Institutos Técnicos Superiores y 45 Institutos de Formación Docente (Banco Mundial, 2018). Ante este escenario, se crea la Agencia Nacional de Evaluación y Acreditación de la Educación Superior (ANEAES) en 2003, a fin de dar respuesta a la necesidad de evaluar la calidad de la educación universitaria, implementándose un proceso sistemático de evaluación con fines de acreditación de las carreras (OEI Paraguay 2019- 2020).

A demás de todo lo expuesto Duarte (2018), destaca que el nivel universitario necesita ser abordado con una gran responsabilidad desde diferentes ámbitos y sectores de cara a los desafíos de una sociedad demandante, con nuevos retos y concepciones tales como la generación del conocimiento al servicio de la sociedad, la investigación e innovación para la transformación de la comunidad circundante y el compromiso con la realidad socio cultural y económica, con sus implicancias en el desarrollo del país, bajo la concepción 
de responsabilidad social y territorial que le compete y sobre todo en estos tiempos de pandemia mundial.

Así mismo, según la OEI Paraguay 2019- 2020, la calidad de la Educación Superior sigue siendo un área de trabajo a seguir mejorando, considerando que la expansión del nivel educativo durante la última década ha sido elevada. Se han creado nuevos centros universitarios, institutos superiores y otras instituciones de formación profesional del tercer nivel.

En este contexto, el proyecto educativo de la universidad debería estar focalizado en dar respuesta a las necesidades de la sociedad, e innovar para mejorar la calidad de vida, con la transformación permanente desde el compromiso con la sociedad para el logro del ascenso de las personas en la pirámide social, con justicia y equidad, apropiación del conocimiento y la innovación para la transformación social (Duarte, 2018).

Conforme a las informaciones abordadas en el presente trabajo se establecen los siguientes objetivos: analizar las estrategias aplicadas por los docentes, tendientes a la transformación de la enseñanza universitaria de calidad y describir la actitud del profesorado hacia la innovación en tiempos de pandemia, teniendo como premisa fundamental que el docente universitario es un profesional con capacidad creativa e innovadora, con habilidad y actitud ante las personas y los hechos, caracterizado por las tres dimensiones presentes en educación: ser, saber y hacer. Dicho de otro modo, actitudes flexibles, dominio de los contenidos y competencia didáctica, capacidades hoy muy necesarias en tiempos de pandemia.

La función social de la universidad en general, en particular la del Paraguay, necesita desarrollar una mirada exhaustiva de sus propios valores, con la convicción firme de buscar la igualdad y el pleno desarrollo de oportunidades para todos, debe buscar la igualdad en el acceso y permanencia, desarrollar capacidades que conlleven una formación universitaria capaz de resolver las problemáticas actuales y coadyuven al desarrollo del entorno, con transformaciones sociales sostenibles, a mediano y largo plazo, para convivir en un espacio donde las personas sean reconocidas por su condición moral, con derechos y libertades, con educación y apropiación de la ciencia, capaces de incidir y modificar las realidades inherentes para el bienestar de la sociedad (Duarte, 2018). 
Hacer realidad lo planteado por Duarte (2018), obliga repensar la función del docente universitario, conforme a lo expuesto por Zabalza, (2004) la docencia en la universidad constituye un espacio de competencias profesionales, dichas competencias en la enseñanza universitaria están compuestas por conocimientos sobre los contenidos que se enseñan y sobre los propios procesos de enseñar y aprender; por ciertas habilidades específicas de comunicación, de manejo de recursos didácticos, de gestión de métodos, de evaluación, además, por un conjunto de actitudes propias de los formadores tales como disponibilidad, empatía, rigor intelectual, entre otros (Zabalza, 2004).

Por todo lo expuesto, es importante reflexionar sobre la gran responsabilidad de las universidades y los docentes que la integran en la contribución a las transformaciones que impactan en la sociedad. De la indagación teórica recogemos además las reflexiones de Grimaldo (2017) respecto a los cambios en el abordaje del conocimiento y la capacidad de innovación social de la universidad, a través de los avances tecnológicos, el intercambio de saberes, la reivindicación de la persona, todos ellos concentrados en la espiral de aprendizaje de una universidad en transición, como menciona el autor (Duarte, 2018).

El acto de enseñar implica siempre una transformación del saber. En el aula, los profesores actúan como hermeneutas o traductores de saberes. A menudo, convierten el saber científico en saber enseñado y tratan de acompañar a los estudiantes en la trasposición didáctica que se produce de una representación a otra. Esta reelaboración, que nosotros hemos denominado transformación del saber, alude a las diversas elecciones que los docentes realizan acerca de cómo encauzar y desarrollar sus explicaciones en el aula (Jarauta, Medina, \& Mentado, 2016).

Es necesario destacar que con la implementación de la educación Virtual en el nivel universitario, exige contar, a su vez, con mecanismos de formación y apoyo en línea al profesorado y a los estudiantes que deberán ser debidamente reforzados. . (UNESCO, 2020). La pandemia obliga a los docentes innovar sus estrategias de enseñanza para lograr aprendizajes, evitar la deserción académica y sobre todo lograr aprendizajes

\section{Actitud del docente universitario para la innovación}

Se considera necesario analizar el enfoque conceptual de innovación, que al decir de López (2017), está relacionado con los procesos desarrollados creativamente al interior de la sala de clase, y también con todos los técnicos, administrativos y académicos 
involucrados con el acto didáctico, un hecho muy significativo las actitudes intencionales que provocan transformaciones profundas que buscan el cambio en el quehacer pedagógico. La innovación supone una transformación, un cambio cualitativo significativo respecto a la situación inicial en los componentes o estructuras esenciales del sistema o proceso educativo. La innovación supone, también, partir de lo vigente para transformarlo (Montalvo, 2011). Implica tener una actitud positiva hacia la innovación, un querer hacer para llegar a hacer.

En este sentido, destaca este autor que para lograr una efectiva innovación se necesita una adecuada formación que posibilite la reflexión al profesorado de su propia práctica y la puesta en marcha de recursos encaminados a trabajar en proyectos cooperativos. La propuesta aquí planteada es caminar hacia la "cultura de innovación", como garante de una mejora de la calidad educativa que asegure innovaciones planificadas y reflexivas, contribuya a iluminar la acción en el aula y un aspecto importante el compromiso de las personas con los procesos de transformación socioeducativa.

Según López la cultura de la innovación se concreta en el marco de tres dimensiones concretas: la científica o el discurso teórico de los especialistas, recogido en los registros textuales y académicos; la administrativa o burocrática, presente en las normativas utilizadas por los gestores del sistema, mediante reglamentos y disposiciones legislativas; $\mathrm{y}$, finalmente, la práctica o la actividad real del profesorado, concretada en una especie de tradición gremial de recursos e instrumentos de apropiación empírica (López, 2017). Otro aspecto muy importante a considerar en la innovación educativa es visualizar la universidad como un sistema organizativo (racional, natural o abierto) donde el logro de la calidad (rendimiento) está determinado tanto por las acciones individuales como por la interacción de los individuos con las unidades organizativas.. En este sentido, amerita analizar también el contexto institucional así como los factores organizativos y ambientales que interactúan en el proceso de enseñanza (Rodríguez, 2001)

En definitiva, lo que se pretende es transitar desde un modelo expositivo, individualista, centrado en la enseñanza del profesor, que transmite la información de forma unidireccional, y que se desarrolla en el espacio del aula; a un modelo constructivista, cooperativo, basado en la interacción, focalizado en el aprendizaje del alumno, que no solo debe conocer sino 
también adquirir conciencia, y que amplía su marco de acción a la realidad social. (Santos Rego, y otros, 2017, p. 32).

La enfermedad del Coronavirus llegó y cambia el escenario de la educación Superior en Paraguay, se respira un aire de incertidumbre, desolación y los docentes deben seguir con las clases, se necesita de creatividad e innovación para responder a los nuevos desafíos y sobre todo a esta crisis a nivel mundial.

Investigadores, docentes, alumnos y el equipo administrativo que da soporte a las actividades académicas han dicho al mundo que la formación no se detiene, que el aprendizaje no se posterga; por lo tanto, el esfuerzo es para todos los actores, la implementación de la educación virtual se impuso repentinamente, estableciéndose migrar a una educación no presencial (Atarama, 2020).

\section{2- ESTRATEGIA METODOLÓGICA}

En la presente investigación, la población de interés o universo constituyó la totalidad de los docentes de la Facultad de Humanidades y Ciencias de la Educación, catedráticos de las diferentes asignaturas de cuatro carreras: Licenciatura en Ciencias de la Educación, Licenciatura en Matemática, Licenciatura en Psicología Educacional y Licenciatura en Psicología Laboral.

Para poner en práctica esta investigación, se procedió a la planificación del mismo, realizando un recorrido teórico de los temas relacionados, se seleccionó el instrumento, un cuestionario construido y validado por Santos Rego, et al., (2017), contextualizado y adaptado conforme a los objetivos de la investigación. Este instrumento contempla dos escalas basadas en un modelo teórico, denominados: práctica docente transformadora, y prácticas docentes innovadoras.

Los integrantes de la población estudiada lo conformaron sesenta y cinco docentes de la Facultad de Humanidades y Ciencias de la Educación de la Universidad Nacional de Pilar, a quienes se les aplicó el instrumento de recolección de datos, abarcando el 100\% de la población en estudio, así mismo se procedió al análisis de los planes de los docentes.

Para la aplicación de los instrumentos se contó con la activa participación de los estudiantes del tercer curso de la carrera Ciencias de la Educación a fin de lograr uno de los objetivos de la carrera "Generar conocimientos científicos que permitan la formación de graduados comprometidos con el contexto socio- político tendiente a propiciar la 
construcción de una educación de calidad" (Facultad de Humanidades y Ciencias de la Educación, 2015, p.11).

El análisis documental, también incorporó la observación, estudio y registro de los planes anuales de los docentes de la Facultad.

El método de investigación que se implementó responde al paradigma cuantitativo, datos cuantificables, fueron aplicados cuestionarios estructurados a todos los docentes de la Facultad, con el propósito de medir las variables, conforme a los objetivos de la investigación, y además se procedió al análisis de las planificaciones anuales de los docentes, con la técnica observación documental. Por ende, la metodología adoptó el enfoque cuantitativo, con diseño observacional; prospectivo; transversal y descriptivo.

\section{3- RESULTADOS Y DISCUSIÓN}

\subsection{Variable: Enfoques de Estrategias aplicadas por el docente}

Para el estudio de esta variable, se ha procedido al análisis y organización de los ítems contemplados en el instrumento, a fin de sistematizar el cuestionario aplicado a los docentes de la Facultad, se contrasta ítems relacionados con el propósito de visibilizar acciones que tienden a la transformación de la educación universitaria con acciones relacionadas al enfoque tradicional de la educación universitaria y estrategias con enfoque constructivistas e innovadoras

Objetivo $\mathbf{N}^{\circ}$ 1: Indagar sobre las estrategias aplicadas por el docente, tendientes a la transformación de la enseñanza universitaria de calidad

Tabla $\mathrm{N}^{\circ} 1$ Presentación de casos prácticos

\begin{tabular}{lccccc}
\hline Ítems & $\begin{array}{l}\text { Nunca } \\
\%\end{array}$ & $\begin{array}{l}\text { Poco } \\
\%\end{array}$ & $\begin{array}{l}\text { Algunas } \\
\text { veces } \%\end{array}$ & $\begin{array}{l}\text { Bastante } \\
\%\end{array}$ & \multicolumn{2}{c}{$\begin{array}{l}\text { Siempre } \\
\%\end{array}$} \\
\hline $\begin{array}{l}\text { 1. Analizo y/o presento casos prácticos } \\
\text { como apoyo para el aprendizaje de los }\end{array}$ & & 15 & 35 & 33 & 17 \\
estudiantes & & & & & \\
\end{tabular}

Fuente: Elaboración propia

Al observar el resultado, se puede deducir que la presentación de casos prácticos, es un enfoque que necesita ser incorporado en mayor porcentaje para lograr el aprendizaje relacionado con la vida real, con planteamientos relativos a la vida actual y evitar de esa forma, la repetición de contenidos alejados de la problemática del entorno.

Es necesario resaltar, según Atarama, (2020), que en la educación virtual, el estudiante, guiado por el profesor, puede utilizar varios recursos que brinda el entorno digital, 
familiar, para acceder a informaciones valiosas, resolver problemas reales y realizar actividades que le permitan afianzar el conocimiento y desarrollar las competencias del perfil de salida de la carrera.

Tabla $\mathrm{N}^{\circ}$ 2: La clase magistral

\begin{tabular}{|c|c|c|c|c|c|}
\hline Ítems & $\begin{array}{c}\text { Nunca } \\
\%\end{array}$ & $\begin{array}{c}\text { Poco } \\
\%\end{array}$ & $\begin{array}{l}\text { Algunas } \\
\text { veces } \%\end{array}$ & $\begin{array}{c}\text { Bastante } \\
\%\end{array}$ & $\begin{array}{c}\text { Siempre } \\
\%\end{array}$ \\
\hline $\begin{array}{l}\text { 2. En mis clases teóricas, virtuales, la clase } \\
\text { magistral es la metodología fundamental. }\end{array}$ & & 12 & 33 & 15 & 40 \\
\hline
\end{tabular}

Fuente: Elaboración propia

Se observa una tendencia de que las clases centradas en la información del docente, la clase magistral, sigue siendo una estrategia implementada en mayor porcentaje a juzgar por los resultados arrojados.

Esta situación se contrapone a lo expresado por Atarama (2020) quien menciona que el alumno siempre ha sido el eje de la educación; sin embargo, en la educación virtual pasa a ser el protagonista indiscutible. En una sesión magistral en la modalidad presencial, la responsabilidad de desarrollar y explicar los contenidos es del profesor; por el contrario, en el modelo de educación virtual, la clase magistral por videoconferencia es un contenido excepcional, lo que predomina es un trabajo de orientación al alumno para ofrecerle mecanismos que le permitan ir explorando la materia.

Con respecto a este punto, amerita resaltar lo expresado por Deeley (2016) quien menciona la necesidad imperiosa en el ámbito universitario de transitar desde un modelo expositivo, individualista, centrado en la enseñanza del profesor, que transmite la información de forma unidireccional, y que se desarrolla en un espacio; a un modelo constructivista, cooperativo, basado en la interacción, focalizado en el aprendizaje del alumno, que no solo debe conocer sino también adquirir conciencia, y ampliar su marco de acción a la realidad social, para lograr efectivamente la transformación del enfoque de enseñanza en el ámbito universitario (Deeley, 2016, como se citó en Santos Rego, y otros, 2017).

Tabla N³: Rol del estudiante

\begin{tabular}{lccccc}
\hline \multicolumn{1}{c}{ Ítems } & Nunca & Poco & Algunas & Bastante & Siempre \\
& $\%$ & $\%$ & veces $\%$ & $\%$ & $\%$ \\
\hline 3. En mi clase virtual el papel de los & 3 & 6 & 13 & 32 & 46 \\
alumnos es estar atentos y tomar apuntes & & & & & \\
\hline
\end{tabular}




\section{conforme a la bibliografía básica del plan}

\section{de estudios.}

Fuente: Elaboración propia

$\mathrm{Al}$ analizar el ítem revela un alto porcentaje de docentes que siguen teniendo preferencias por las estrategias que garanticen la supremacía del profesor. La literatura analizada sobre las estrategias de enseñanza en la educación superior conducen a destacar los aportes de Duarte (2018), respecto al rol de la enseñanza actual considerando los desafíos de una sociedad demandante, con nuevos retos y concepciones tales como la generación del conocimiento al servicio a la sociedad, la investigación e innovación de cara a la transformación de la comunidad circundante y el compromiso con la realidad socio cultural y económica, con sus implicancias en el desarrollo del país.

Tabla $\mathrm{N}^{\circ} 4$ Aplicación de estrategias innovadoras

\begin{tabular}{llllll}
\hline Ítems & $\begin{array}{l}\text { Nunca } \\
\%\end{array}$ & $\begin{array}{l}\text { Poco } \\
\%\end{array}$ & $\begin{array}{l}\text { Algunas } \\
\text { veces \% }\end{array}$ & $\begin{array}{l}\text { Bastante } \\
\%\end{array}$ & $\begin{array}{l}\text { Siempre } \\
\%\end{array}$ \\
\hline 4. En mi clase virtual planifico y ejecuto & 51 & 24 & 19 & 4 & 2 \\
aprendizajes basados en juegos: & & & & &
\end{tabular}

Gamificación, microlearning.

Fuente: Elaboración propia

Al realizar una mirada crítica el resultado de la Tabla $\mathrm{N}^{\circ} 4$, se observa la poca implementación de estrategias metodológicas didácticas acordes a la utilización de las tecnologías. Ciertamente que la suspensión de clases implica en forma urgente de una experiencia, preparación, actualización en la docencia virtual, demandando unas competencias tecnológicas a los docentes y una rápida adecuación y adaptación de las planificaciones a fin de responder a las nuevas tendencias de la Pedagogía del siglo XXI, como ser la implementación de la Gamificación o microlearning como estrategias de enseñanza y aprendizaje.

Es así que con la pandemia instalada a nivel mundial amerita según Atarama (2020) implementar una educación virtual enmarcada en una cultura de la convergencia y del compartir informaciones donde el profesor señale como material de la asignatura el uso de los diversos recursos formativos e informativos gratuitos que se encuentran en el entorno digital, desde un artículo científico hasta cursos completos, y luego llevar a un debate y un análisis en el aula virtual. 
Tabla N5: Estrategias que fomentan el pensamiento crítico

\begin{tabular}{llllll}
\hline Ítems & $\begin{array}{l}\text { Nunca } \\
\%\end{array}$ & $\begin{array}{l}\text { Poco } \\
\%\end{array}$ & $\begin{array}{l}\text { Algunas } \\
\text { veces } \%\end{array}$ & $\begin{array}{l}\text { Bastante } \\
\%\end{array}$ & $\begin{array}{l}\text { Siempre } \\
\text { \% }\end{array}$ \\
\hline $\begin{array}{l}\text { 5. Promuevo actividades que fomentan el } \\
\text { pensamiento crítico y reflexivo de los }\end{array}$ & & & & & \\
alumnos. & & & & & \\
\end{tabular}

Fuente: Elaboración propia

Como se puede observar, las estrategias utilizadas por los docentes para el desarrollo de las clases, tienden a valorar estrategias tradicionalistas, antes que instalar actividades que promuevan el pensamiento crítico.

El desarrollo del pensamiento crítico en la educación superior adquiere particular importancia de los futuros profesionales, pues permite que los estudiantes desarrollen competencias y capacidades que les favorezcan el análisis crítico y puedan encontrar soluciones prácticas y efectivas (Palacios, Álvarez, Moreira, \& Morán, 2017).

Santos Rego (2016), por su parte, expone que la Universidad debe formar ciudadanos responsables, críticos y participativos, además de buenos profesionales, en el contexto de una sociedad del conocimiento sometida a rápidos y profundos cambios.

\subsection{Variable: Actitud del profesorado hacia la innovación en tiempos de pandemia}

Objetivo $\mathbf{N}^{\circ}$ 2: Describir la actitud del profesorado hacia la innovación

Tabla $N^{\circ} 6$ Actitud de aceptación de metodologías con enfoque social

\begin{tabular}{llllll}
\hline Ítems & $\begin{array}{l}\text { Nunca } \\
\%\end{array}$ & $\begin{array}{l}\text { Poco } \\
\%\end{array}$ & $\begin{array}{l}\text { Algunas } \\
\text { veces } \%\end{array}$ & $\begin{array}{l}\text { Bastante } \\
\%\end{array}$ & $\begin{array}{l}\text { Siempre } \\
\%\end{array}$ \\
\hline $\begin{array}{l}\text { 6. Demuestro interés en actividades } \\
\text { que promueven metodologías de }\end{array}$ & & 35 & 22 & 12 & 13 \\
resolución de problemas del entorno & & & & & \\
social, con un enfoque reflexivo & & & & & \\
\hline
\end{tabular}

Fuente: Elaboración propia

$\mathrm{Al}$ analizar la actitud del profesorado hacia estrategias participativas y con enfoque social, se visualiza en los resultados una tendencia significativa hacia modos conductistatradicional de concebir la práctica educativa, centrado en la enseñanza del profesor, en este sentido Santos Rego, et. al (2017) destacan la importancia de implementar estrategias que promuevan la implementación de proyectos que impliquen el aprendizaje servicio, donde los alumnos "aprenden mientras actúan sobre necesidades reales del entorno con 
la finalidad de mejorarlo" (p.39),por lo tanto, se observa una tendencia de mayor de inclinación de los docentes que omiten aplicar estrategias, metodologías de resolución de problemas del entorno social, con un enfoque reflexivo.

En este sentido Atarama (2020) señala que en la educación superior con la pandemia en auge, se instala la educación virtual activa, donde el rol y la actitud del profesorado deben incluir en su planificación procesos de aprendizajes que permitan al estudiante desarrollar las competencias y capacidades del curso. El profesor ha de ser capaz de facilitar y favorecer el uso de ecosistemas de los medios, como ser las redes sociales y los entornos colaborativos digitales. Utilizar las plataformas digitales interactivas y las actividades en red con una finalidad educativa puede ser una estrategia muy acertada en la educación superior.

Tabla $\mathrm{N}^{\circ}$ 7. Actitud de aceptación del aprendizaje autónomo

\begin{tabular}{lllllll}
\hline Ítems & $\begin{array}{l}\text { Nunca } \\
\%\end{array}$ & $\begin{array}{l}\text { Poco } \\
\%\end{array}$ & $\begin{array}{l}\text { Algunas } \\
\text { veces } \%\end{array}$ & $\begin{array}{l}\text { Bastante } \\
\%\end{array}$ & $\begin{array}{l}\text { Siempre } \\
\%\end{array}$ \\
\hline $\begin{array}{l}\text { 7.Promuevo actividades tendientes al } \\
\text { aprendizaje autónomo de los }\end{array}$ & & & & & 12 & 19 \\
estudiantes
\end{tabular}

Fuente: Elaboración propia

Con relación a la actitud del docente de promover el aprendizaje autónomo, la mayor tendencia observada implica que aún falta incentivar en la actitud del educador reflexionar sobre la importancia del aprendizaje autónomo.

Cabe resaltar la importancia de la metacognición, como la capacidad de autorregular los procesos de aprendizaje, que permite a los alumnos a ser autónomos en su aprendizaje y a mantener una actitud crítica sobre la información, el conocimiento y sobre sus propias estrategias de aprendizaje (Mosquera, 2019) Más aún con esta pandemia, y con las clases virtuales, se podrían vivenciar actividades que permitan indagar, investigar, conocer más, desarrollar la responsabilidad del estudiante en la gestión de su aprendizaje La metacognición puede ser desarrollada y practicada para, contribuir a la autonomía de los estudiantes. Por lo tanto, para lograr innovar permanentemente el docente debe ser capaz de evaluar la propia forma de enseñar y de aprender para poder ayudar a los estudiantes a desarrollar esas estrategias (Mosquera 2019). Según este autor es una manera de mantener viva la llama de la innovación en el actuar docente 
Actualmente existe mayor complejidad y exigencia intelectual tanto para docentes universitarios como para estudiantes de este nivel, en el sentido de que la sociedad actual exige pasar de una visión bancaria que apuntaba a la reproducción y transmisión de la información a otra visión que apunte a la transformación de la información en conocimiento y a la acción de un liderazgo positivo en la sociedad del entorno de influencia de la Universidad (Donoso, 2018). Con la adaptación de los nuevos modelos pedagógicos orientados en dotar a los estudiantes de conocimientos que le garanticen una formación permanente y continua, de esta forma las universidades estarían orientando a los estudiantes a una formación basada en una educación permanente, continua y con pertinencia social (Prieto, Mijares, \& Llorent, 2014).

Según Vera \& Mazadiego (2010), se necesita fomentar la reflexión en el docente en su quehacer áulico, que le permita participar en la formación de estudiantes más competentes y abiertos al cambio para que al egresar puedan ser competitivos, con la ayuda de actitudes llenas de autocontrol y dinamismo, siempre bajo el objetivo de ser un facilitador del aprendizaje, más que un guía, ser un mediador que conciba al aprendizaje como una actividad social y de experiencia compartida, cuya sustentabilidad sea el trabajo cooperativo, en al afán de provocar aprendizajes significativos acordes a la realidad social de cada uno de sus estudiantes

Tabla $N^{\circ} 8$ Actitud hacia la utilización de plataformas interactivas

\begin{tabular}{llllll}
\hline Ítems & $\begin{array}{l}\text { Nunca } \\
\%\end{array}$ & $\begin{array}{l}\text { Poco } \\
\%\end{array}$ & $\begin{array}{l}\text { Algunas } \\
\text { veces } \%\end{array}$ & $\begin{array}{l}\text { Bastante } \\
\%\end{array}$ & $\begin{array}{l}\text { Siempre } \\
\%\end{array}$ \\
\hline $\begin{array}{l}\text { 8. Me gusta planificar e implementar } \\
\text { estrategias activas }\end{array} \quad$ utilizando & & & 24 & 11 & 32 \\
plataformas interactivas, como & & & & & \\
herramienta didáctica con técnicas & & & & & \\
dinámicas &
\end{tabular}

Fuente: Elaboración propia

La actitud de preferencia hacia la planificación de metodologías innovadoras con la utilización de estrategias didácticas interactivas es en bajo porcentaje, al analizar los planes anuales también se observan un elevado porcentaje de dinámicas tendientes a lo tradicional.

La pandemia ha llegado de sorpresa, instalando la necesidad de clases virtuales, interactivas, dinámicas, cuyas características principales de las planificaciones docentes 
deben tender a que el estudiante se mantenga activo y profundice los conocimientos en forma independiente (Castro, 2020).

Es importante mencionar que para las clases virtuales el aprendizaje interactivo permite enfocar a la inteligencia del estudiante en forma integral, por lo que no solo es importante el desarrollo intelectual, sino también las habilidades artísticas, culturales, la autorregulación, elementos que apuntan a la formación integral del dicente (Castro, 2020).

Cobra real trascendencia, por lo tanto, la incorporación efectiva de los entornos virtuales e interactivos del aprendizaje; donde los docentes tienen la posibilidad de mejorar sus prácticas de aula, crear entornos de aprendizajes más dinámicos e interactivos en el proceso de enseñanza y aprendizaje de los estudiantes, facilitar el trabajo en equipo y el cultivo de actitudes sociales (Vence, 2014)

\section{CONCLUSIÓN O CONSIDERACIONES FINALES}

El abordaje de la caracterización de la transformación de la enseñanza universitaria y la actitud del profesorado hacia la innovación, destaca la necesidad de una innovación profunda del accionar de los docentes universitarios por la permanente evolución de los conocimientos, los cambios vertiginosos que impuso la pandemia y la necesidad de un enfoque sustentable y social de la educación. (Vainsencher, 2018), ratifica que las características de un buen docente es su compromiso por enseñar, infundir confianza y motivar para el aprendizaje, comprender que las aulas son heterogéneas, los cambios son constantes y los estudiantes no son seres aislados sino que forman parte de un contexto cultural, social, familiar, económico y político.

En este sentido, las estrategias de enseñanza caracterizadas en este trabajo demuestran la necesidad de implementar sistemáticamente capacitaciones de apoyo teórico-práctico a los docentes universitarios, con el firme propósito de incorporar metodologías activas, interactivas y variadas en los procesos de las clases virtuales, concretar la motivación y apoyo de acercamiento al estudiante para el aprendizaje autónomo. La pandemia obligó a los actores académicos implementar el uso de las clases virtuales, exigiendo por ende, estar en la vanguardia de elementos tecnológicos y pedagógicos que permitan crecer y desarrollara las competencias establecidas en el perfil de salida de cada carrera

De la misma manera, la actitud del profesorado hacia la innovación requiere ser estimulada, a fin de implementar cambios significativos que favorezcan el aprendizaje 
interactivo, colaborativo, la metacognición en la forma de enseñar, la investigación digital como nueva forma de enseñar y aprender, demostrando ser un docente reflexivo y selectivo de las informaciones.

Se concluye resaltado que es de vital importancia la actitud positiva del docente hacia la innovación, no existe otro camino para mejorar el accionar profesional acorde a la crisis mundial. Una actitud positiva permite el crecimiento profesional, logra mejores resultados y ayuda a enfrentar esta situación originada por la pandemia.

\section{LISTA DE REFERENCIAS}

Atarama, T. (05 de abril de 2020). http://udep.edu.pe/hoy/2020/la-educacion-virtual-entiempos-de-pandemia. Obtenido de www.udep.edu.pe

Castro, M. (23 de 06 de 2020). https://lamenteesmaravillosa.com/de-que-trata-el-aprendizajeinteractivo/. Obtenido de https://lamenteesmaravillosa.com/de-que-trata-el-aprendizajeinteractivo/

Donoso, S. (2018). Nuevo rol del docente, nuevos desafíos de la docencia. Talca: Instituto de Investigación y desarrollo educacional.

Duarte, M. (2018). Las universidades en Paraguay y su influencia en la transformación social. Revista científica de la UCSA Volumen $5 N^{\circ} 3,64-68$.

Henríquez Guajardo, P. , P. (2018). Conferencia Regional de Educación Superior. Res'menes ejecutivos, 111-133.

Jarauta, B., Medina, J., \& Mentado, T. (2016). La transformación del saber en la enseñanza universitaria. Revista de investigación educativa, 471-485.

López, R. (2017). Hacia una innovación docente de calidad en la educación superior. Foro educacional $N^{\circ} 28,11-28$.

Montalvo, J. (2011). Innovación en la educación. Anuario jurídico y económico, 567-582.

Mosquera, I. (2019). Metacognición. Promover el aprendizaje autónomo de los estudiantes. Revista UNIR.

OEI. (14 de 03 de 2020). https://oei.org.py/acercade/programa-pais. Obtenido de https://oei.org.py/acercade/programa-pais

Palacios, W., Álvarez, M., Moreira, J., \& Morán, C. (24 de 04 de 2017). Una mirada al pensamiento crítico en el proceso docente educativo de la educación superior.

Obtenido de http://www.scielo.sa.cr/pdf/aie/v14n2/a20v14n2.pdf: http://www.scielo.sa.cr/pdf/aie/v14n2/a20v14n2.pdf

Prieto, M., Mijares, B., \& Llorent, V. (2014). Roles del docente y del alumno universitario desde la perspectivas de ambos protagonistas del hecho educativo. Revista electrónica de Humanidades, Educación y Comunicación Social, 1-8. 
Santos Rego, M., Sotelino, M., Jover, G., Naval, C., Álvarez, J., \& Vázquez, V. (2017). Diseño y validación de un cuestionario sobre práctica docente y actitud del profesorado universitario hacia la innovación. Revista Educación XXI. Vol 20 N², 39-71.

UNESCO. (02 de 01 de 2019). Mecanismos regionales. Obtenido de https://es.unesco.org/themes/educacion-2030-ods4/mecanismos-regionales: https://es.unesco.org/themes/educacion-2030-ods4/mecanismos-regionales

UNESCO. (2 de abril de 2020). EL CORONAVIRUS COVID-19 Y LA EDUCACIÓN SUPERIOR: IMPACTO Y RECOMENDACIONES. Obtenido de https://www.iesalc.unesco.org/2020/04/02/el-coronavirus-covid-19-y-la-educacionsuperior-impacto-y-recomendaciones/: www.unesco. org.com

Vainsencher, A. (2018). El docente como facilitador del aprendizaje . Reflexión Académica $X X X V, 1-9$.

Vence, L. (2014). USO PEDAGÓGICO DE LAS TIC PARA EL FORTALECIMIENTO DE ESTRATEGIAS DIDÁCTICAS DEL PROGRAMA TODOS A APRENDER DEL MINISTERIO DE EDUCACIÓN DE COLOMBIA. Congreso Iberoamericano de Ciencia, Tecnología, Innovación y Educación, 1-21.

Vera, A., \& Mazadiego, T. (2010). Una perspectiva sobre las actitudes y el deber ser de los docentes. revista de Educación y Desarrollo, 53-58.

Villafuerte, P. (2020). Educación en tiempos de pandemia. Observatorio de Innovación Educativa, 1-5.

Villalba Benítez, E. (2017). Educación Superior en Paraguay: análisis de la inversión estatal en Universidades Públicas entre 2007 al 2016. En N. P. (Compiladora), Jornadas de Investigación en Educación Superior (págs. 123 - 146). Montevideo: Universidad de la República de Uruguay.

Wolpert, R. (2019). Foro internacional. México: Vanguardia en la educación.

Zabalza, M. (2004). Innovación en la enseñanza univesritaria. revista de educación: Contextos educativos, 113-136. 